\title{
La marée noire vue du bureau ovale : processus rhétoriques pour conceptualiser la crise du golfe du Mexique (avril 2010)
}

\section{Stéphanie Bonnefille}

\section{(2) OpenEdition \\ Journals \\ Édition électronique \\ URL : http://journals.openedition.org/esa/1647 \\ DOI : 10.4000/esa. 1647 \\ ISSN : 2650-2623 \\ Éditeur \\ Société de stylistique anglaise}

Édition imprimée

Date de publication : 31 décembre 2011

Pagination : 131-146

ISSN : 2116-1747

\section{Référence électronique}

Stéphanie Bonnefille, "La marée noire vue du bureau ovale : processus rhétoriques pour

conceptualiser la crise du golfe du Mexique (avril 2010) », Études de stylistique anglaise [En ligne],

3 | 2011, mis en ligne le 27 novembre 2018, consulté le 30 avril 2019. URL : http://

journals.openedition.org/esa/1647; DOI : 10.4000/esa.1647 


\title{
LA MARÉE NOIRE VUE DU BUREAU OVALE : PROCESSUS RHÉTORIQUES POUR CONCEPTUALISER LA CRISE DU GOLFE DU MEXIQUE (AVRIL 2010)
}

\author{
Stéphanie Bonnefille \\ Université Michel de Montaigne, Bordeaux 3 - CLIMAS EA 4196
}

\begin{abstract}
This paper follows on from the investigations conducted in three previous pieces of research (Bonnefille 2008, 2009 et 2011b). Based on a framework that was previously defined as cognitive rhetoric (i.e. a selected combination of tools coming from cognitive linguistics and rhetoric), the author analyzes the speech given by President Obama on the oil spill, which occurred in the Gulf of Mexico in 2010. The paper focuses on the processes of conceptualization at work during the worst oil spill in American history.
\end{abstract}

Keywords: cognitive rhetoric, applied cognitive linguistics, narrative framing, ecospeak, American presidential speeches, Obama, energy crisis, oil spill

A study of style (...) is an assistance to a rational statement of what society is doing with its language. (Darbyshire 1971, 46)

\section{Introduction}

Le 20 avril 2010, dans le golfe du Mexique, la plate-forme pétrolière Deep Water Horizon exploitée par la société British Petroleum a explosé puis coulé par mille cinq cents mètres de fond. Les techniques diverses employées pour mettre fin à la marée noire se sont succédées : colmatage, injection de

1 Cet article, accepté par le comité de lecture, est issu de la communication présentée en mai 2011 dans l'atelier de stylistique lors du $51^{\mathrm{e}}$ congrès de la SAES aux universités Paris Diderot et Paris Sorbonne Nouvelle. 
boue, pose d'un couvercle géant, pompage, incendies contrôlés. Mais au fil des semaines, le pétrole a continué de se répandre dans le golfe. L'administration américaine et les experts indépendants ont divergé sur la quantité de pétrole qui s'est déversé jour après jour : en mai, selon la première, cinq mille barils ${ }^{2}$ par jour, contre vingt à quarante mille par jour depuis le début de la catastrophe d'après les seconds, soit la contenance de 1,5 à 2,5 bassins olympiques.

Après une première visite sur site le 2 mai 2010 et plusieurs interventions télévisées informelles souvent jugées approximatives par les médias américains et internationaux, le président Obama a prononcé, le 15 juin 2010, son premier discours officiel en direct du bureau ovale ${ }^{3}$ au sujet de cette catastrophe pétrolière sans précédent et immédiatement déclarée « catastrophe nationale $»^{4}$. Pendant dix-sept minutes, le président s'est adressé à la nation en ne cessant de souligner la forte implication de l'administration, la prise en compte des victimes et des dégâts, la gestion des conséquences à moyen et à long termes sur l'environnement et sur l'économie, la volonté de mettre BP face à ses responsabilités. Afin de capter ${ }^{5}$ la confiance de l'auditoire, et plus particulièrement celle des citoyens directement concernés d'un point de vue géographique ${ }^{6}$ et encore sous le choc des dégâts causés par les ouragans Katrina et Rita en août et en septembre 2005, le discours met en avant la nécessité d'adopter une politique environnementale nouvelle qui doit impérativement s'orienter vers les énergies dites «propres».

L'objet de cet article, qui s'inscrit dans le cadre théorique de la rhétorique cognitive (voir l'élaboration de ce terme dans Bonnefille 2011b), est de mettre en lumière les processus rhétoriques les plus prégnants dans ce discours politique afin de déterminer la logique de communication et, plus largement, la manière dont la Maison-Blanche façonne la crise environnementale dans le but d'en maîtriser la conceptualisation. Nous prendrons le terme "rhétorique » dans son acception aristotélicienne et non stoïcienne, c'est-à-dire «l'art de persuader » ou le désir de l'emporter, et non celui de «bien dire». Nous y allierons des outils issus de la linguistique cognitive qui permettent d'analyser les mécanismes cognitifs sous-jacents à la construction de la représentation de cette catastrophe environnementale.

2 Un baril contient cent soixante litres de pétrole.

3 http://www.whitehouse.gov/the-press-office/remarks-president-nation-bp-oil-spill

4 En avril 2010, en déclarant la marée noire «catastrophe nationale », Janet Napolitano, secrétaire à la Sécurité intérieure des États-Unis, a permis le déploiement maximal de secours à disposition dans le pays, et notamment celui de l'armée.

5 Le terme est ici pris dans l'acception qui lui est attribuée en analyse de discours, «captation » signifiant, entre autres, la stratégie mise en place afin de séduire l'interlocuteur (Maingueneau 1996, 23).

6 Selon des sources informelles provenant des États-Unis, il semble que ce discours officiel soit passé totalement inaperçu pour une grande majorité de la population. 


\section{La disposition du discours}

Le discours se divise en six parties distinctes qu'il convient de détailler afin d'en faire ressortir la disposition (Gardes-Tamine 1996, 96-97) qui s'avère extrêmement travaillée. La transcription du discours correspond à deux cent une lignes de texte. Les parts du diagramme présenté ci-dessous sont calculées sur la base de ce nombre de lignes qui correspond donc à $100 \%$.

La première partie (i), l'exorde, rappelle brièvement les divers défis auxquels les États-Unis sont actuellement confrontés : la récession économique, le combat contre Al-Qaeda à l'étranger et la marée noire dans le golfe. Vient ensuite l'exposition rapide des faits (ii) suivie des solutions et des actions déjà adoptées tout autant que de celles qui restent à mettre en œuvre (iii). Le président détaille alors le plan d'action dans la partie la plus longue de son discours (iv), elle-même sous-divisée en trois points qui sont introduits par les expressions explicitement structurantes : «First, the cleanup » (1. 33), «That's why the second thing we're focused on is the recovery and restoration of the Gulf Coast» (1. 58) et « The third part of our response plan is the steps we're taking to ensure that a disaster like this does not happen again » (1. 86-87). En d'autres termes, les trois volets de ce plan sont : 1 . Nettoyer, 2 . Reconstruire et 3. S'affranchir du pétrole pour que jamais plus ce type de catastrophe ne se reproduise. Le discours s' oriente ensuite vers la deuxième partie la plus longue du discours (v), celle qui est consacrée au discours environnemental et à la mise en exergue de l'addiction du peuple américain au pétrole. Le président plaide pour une politique nouvelle qui favorise l'exploitation d'énergies propres dans tous les secteurs de l'industrie. Ce type de discours, nous le verrons, est aussi appelé ecospeak. Enfin, le discours s'achève sur un épilogue (vi) orienté vers le révolu (les grands défis déjà accomplis par les États-Unis) tout autant que vers l'avenir et l'espoir (le pays dans lequel les citoyens veulent voir leurs enfants grandir). Cette dernière partie comporte également une morale religieuse dont nous dégagerons l'effet produit sur l'auditoire.

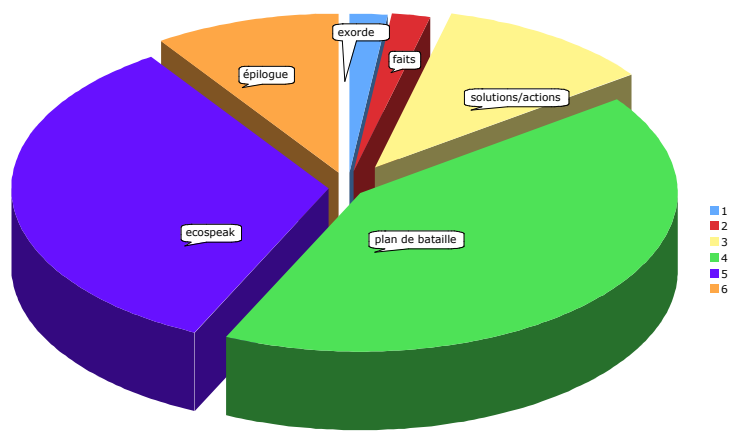


Bien que l'analyse renvoie à toutes les parties présentées, nous consacrerons une étude plus poussée aux parties intitulées «plan de bataille » et « épilogue » d'une part (section 3) et «ecospeak» de l'autre (section 4).

\section{Le scénario de la guerre}

Lors d'un précédent travail (Bonnefille 2009) pour cette même revue, nous avions présenté la notion de cadrage narratif («narrative framing ») telle qu'elle est définie et résumée par Cox $(2006,186)$ :

Narrative framing refers to how the media organize the bits and facts of phenomena through stories to aid audiences' understanding, and to the potential for this organization to affect our relationships to the phenomena being represented.

Selon ce spécialiste de la communication environnementale, le «cadre » en question correspond à une carte cognitive d'interprétation utilisée par le sujet afin d'organiser ses expériences en connaissances cohérentes et réutilisables. Le concept de frame, ainsi que ses origines, est longuement exploré dans Bonnefille 2008 et Bonnefille 2009. Or, comme le rappelle très simplement Maingueneau $(1976,71)$ bien avant $^{7}$ Cox, par exemple, et bien avant Salmon (2007), « [1]a narration est loin de concerner seulement l'histoire ou les romans, elle définit également un ensemble de textes non littéraires et quotidiens ».

Le discours à l'étude repose en effet sur un cadrage narratif particulier, ou processus de «storytelling » (Salmon 2007), qui permet à l'orateur de délimiter les contours et le contenu d'un récit. Notons que des études analogues sont également menées en sciences politiques. Ritaine (2010) démontre que le phénomène d'immigration qui a lieu actuellement en Italie est construit, par le gouvernement, sur la base de «scripts » permettant aux citoyens de raisonner grâce à des représentations narratives simples mais dont le pouvoir de manipulation rend l'écart entre représentation et réalité toxique pour la démocratie italienne.

\section{L'intrigue : les États-Unis en guerre}

La narration sous-tend ici la stratégie de communication adoptée. En effet, le discours ne relate pas les faits de manière factuelle. Et comment le pourrait-il ? Le président doit avant tout capter l'attention de l'auditoire, frapper son esprit, ses sens et son cœur, ou encore «exciter ses passions »

7 Nous n'oublierons pas les travaux fondateurs de Propp, de Barthes, de Ricœur et de Genette dans le domaine de la mise en récit et des arcanes de la narration. 
(Aristote 1991, 83). Aussi, dès l'exorde, le discours repose-t-il sur le cadrage narratif de la guerre que doit soudainement mener le gouvernement américain contre la marée noire :

1. (...) [T] speak with you about the battle we're waging against an oil spill that is assaulting our shores and our citizens. (1. 5-6)

L'isotopie de la guerre est en effet présente tout au long du discours et l'implication de l'armée dans cette crise (voir la note de bas de page $\mathrm{n}^{\circ} 4$ ) ne fait que renforcer cette métaphore conceptuelle (Lakoff 1980) GERER LES DOMMAGES CAUSES PAR LA MAREE NOIRE EST FAIRE LA GUERRE :

2. We will fight this spill (1. 26) ; our battle plan (1. 30) ; thousand of ships and vessels are responding in the Gulf (1. 37-38); the deployment of over 17, 000 National Guard members (1. 38-39) : these troops (1. 42); before this siege is done (1. 57); that living is now in jeopardy (1. 61)

Mais quels sont alors les éléments constitutifs d'un tel scénario ? La guerre comprend, en général, un ennemi à combattre, des victimes à sauver, un dirigeant soutenu par la confiance de la nation, un plan de bataille, une armée, des moyens technologiques, un chant, un hymne ou des mythes qui ravivent le sentiment d'unité et le patriotisme. Or le discours à l'étude prend effectivement appui sur une distribution de rôles prototypiques, sur un plan de bataille ou encore sur un texte censé se faire l'écho de la morale de cette guerre métaphorique.

\section{Distribution : l'ennemi, le sauveur, les victimes}

L'ennemi prend tour à tour les traits du pétrole et ceux de BP. Est également tenue pour responsable l'agence fédérale américaine Minerals Management Service qui est censée réguler l'exploitation des énergies fossiles sur le sol américain et délivrer des permis de forage de manière raisonnée :

3. We will make BP pay for the damage their company has caused. (1. 27) ; (...) I will meet with the chairman of BP and inform him that he is to set aside whatever resources are required to compensate the workers and business owners who have been harmed as a result of this company's recklessness. (1. 68-69); Over the last decade, (Minerals Management Service) has become emblematic of a failed philosophy (...) that says corporations should be allowed to play by their own rules and police themselves (1. 104$06) ;(\ldots)$ to cleanup the worst of the corruption at this agency. (1.111)

La lutte dont Obama fait état est acharnée et sera probablement longue. Elle implique, dans un climat d'urgence, le déploiement de forces militaires, de vaisseaux, de troupes : 
4. 30000 personnel who are working (1. 36) ; Thousands of ships and other vessels are responding in the Gulf (1. 37-38); the deployment of over 17, 000 National Guard members (39).

Obama constitue son armée en s'entourant de personnes proches dont nous apprenons le titre, le nom, la fonction présentée comme prestigieuse ou une information valorisante :

5. [A] team led by Dr. Steven Chu, a Nobel Prize-winning physicist and our nation's Secretary of Energy (1. 14); an effort led by Admiral Thad Allen, who has almost 40 years of experience responding to disasters (1. 35) ; I asked Ray Mabus, the Secretary of the Navy, a former governor of Mississippi, and a son of the Gulf (1. 81-82); When Ken Salazar became my Secretary of Interior (1. 110) : Michael Bromwich, who was a tough federal prosecutor and Inspector General (1. 114).

Les victimes, tout autant que l'ennemi d'un côté et que «le sauveur » et son armée de l'autre, sont nommées: ce sont les habitants du Golfe, les pêcheurs, et parfois l'économie personnifiée :

6. (...) [W]e will do whatever's necessary to help the Gulf Coast and its people recover from this tragedy. (1. 27-29) ; (...) to help our neighbors in the Gulf (...) (1. 31/182).

Afin de renforcer l'adhésion de l'auditoire, le discours fait même usage de l'outil de personnalisation ${ }^{8}$ pour mettre en avant certains personnages précis dans une volonté de proximité et de dramatisation:

7. I've talked to shrimpers and fishermen (...) (1. 61-62) ; I've talked to owners of shops and hotels (...) (1. 64) ; the families I met (...) (1. 92)

Ce segment de discours s'apparente alors à une sorte de micro-trottoir conduit par le président lui-même.

\section{Plan de bataille et sécurité}

Le plan de bataille («the battle plan », 1. 30) s'articule en trois volets selon une chronologie logique : l'opération de nettoyage («the cleanup»), sur laquelle nous reviendrons dans la quatrième partie, la reconstruction ( « the Gulf Coast Restoration plan », 1. 83), puis la mise en place de moyens permettant d'éviter qu'une telle catastrophe ne se reproduise. En effet, la création d'une commission nationale (1.94) définira les causes de cette catastrophe et travaillera à la mise en place de nouvelles normes de sécurité. Afin de soutenir le travail d'investigation de cette commission en matière de sécurité, le président a imposé un moratoire de six mois sur le forage en eaux profondes.

8 Traduction du terme « characterization », (Toolan 1988, 97-99 et 99-102). 
Soulignons que la notion de sécurité revient à plusieurs reprises dans le discours sous diverses formes (adjectifs et substantifs) :

8. Absolutely safe (offshore drilling) (1. 89) ; additional safety and environmental standards (1. 95) ; for the sake of (the) safety ( of the people who work on these rigs) (1. 98) ; safety inspections (1. 109); better safety standards (1.118).

La thématique de la sécurité est essentiellement associée au forage et permet au président de faire valoir sa prise de responsabilité par rapport à la catastrophe. Toutefois, elle survient également dans deux autres cas. Par contiguïté sémantique, le président opère en effet un glissement de "safety » vers « security » pour y joindre l'adjectif «national» (1. 161). Ainsi, il rappelle à ses compatriotes qu'il veille également à la sécurité sur le sol américain. Il active, de fait, la thématique antagoniste: celle de l'insécurité diffuse qui génère la peur et renforce la posture protectrice de l'administration. Lakoff (2004) parlerait ici de l'activation d'un cadre de connaissances (ou «frame») effectué par le seul emploi d'une expression. Le linguiste illustre ce phénomène cognitif, dont la puissance en terme de stratégie discursive est non négligeable, en prenant l'exemple d'un groupe d'étudiants à qui il interdit de penser à un éléphant. L'exercice s'avère impossible car le simple fait de parler de l'éléphant génère à l'esprit, aussi évanescent qu'il puisse être, un réseau de connaissances, de mythes et de représentations prêts à l'emploi. Nous soulignerons que les jalons de ce message implicite sont posés dès le début du discours et que l'emploi de l'adverbe «wherever» le pousse à sa puissance maximale :

9. Abroad, our brave men and women in uniform are taking the fight to al Qaeda wherever it exists. (1. 3-4).

Nous pouvons nous demander pourquoi, dans un discours de soutien face aux citoyens touchés par la crise, il est nécessaire de prendre la guerre contre Al-Qaida comme point de départ. Cette référence, qui active le cadre même de la guerre contre le terrorisme - et plus largement les événements du 11Septembre qui ont déclenché le programme War on Terror-, permet à Obama, deux lignes plus bas, de s'installer dans cette dynamique offensive, donc réactive et a priori rassurante : «the battle we're waging against an oil spill » (1. 5). La deuxième occurrence apparait lors de la référence à Neil Armstrong : « (...) our ability (...) to land a man safely on the surface of the moon. » (1. 175). En faisant appel à la mémoire collective, Obama ravive la flamme du sentiment national tout en rappelant que les États-Unis se construisent précisément dans des défis comme celui qui se présente à eux. En outre, la notion de sécurité permet de rappeler que ces défis sont toujours relevés dans le respect de la sécurité des citoyens américains sur leur sol, ou ailleurs. 


\section{Patriotisme et morale : images de mémoire et analogie}

Il est une catégorie d'images dont il n'est que rarement fait mention en ces termes dans une analyse de discours politique : les «images de mémoire » (Gardes-Tamine 1996, 39-44) dont la puissance évocatrice permet, souvent par le biais d'un choc émotionnel, d'unir une nation au sein d'une même histoire :

10. The one answer I will not settle for is the idea that this challenge is too big and too difficult to meet. You see, the same thing was said about our ability to produce enough planes and tanks in WWII. The same thing was said about our ability to harness the science and technology to land a man safely on the moon. (1. 171-75)

La seconde guerre mondiale, qui renforce encore le cadre narratif de la guerre évoqué plus haut, de même que l'alunissage et les premiers pas sur la Lune constituent deux grands repères mémoriels dans l'histoire des États-Unis et rappellent aux citoyens que la guerre contre cette marée noire tout autant que l'orientation de la nation vers les énergies «propres » constituent donc un défi qui peut être relevé. D'autres termes clés de la chronologie des États-Unis sont également mentionnés : Al-Qaida, les ouragans Katrina et Rita ou l'expression «what has defined us as a nation since our founding» (1. 177), dont le substantif «founding » active la mémoire des pères fondateurs. Sont ici évoquées des catastrophes qui ont été surmontées : le 11-Septembre 2001 et les deux ouragans qui se sont abattus, coup sur coup, sur les États-Unis quatre ans plus tard. Obama se réfère également à la mythologie de son pays. Ainsi, comme l'énonce Gardes-Tamine (1996, 43),

(...) la mémoire, ce n'est pas seulement pour l'orateur la nécessité de se remémorer les éléments, objets, êtres, actions dont il doit parler, et les mots les plus adaptés pour en parler, c'est aussi la volonté de frapper l'imagination pour graver le discours dans l'esprit des auditeurs. La mémoire au sens restreint de partie de la rhétorique s'appuie donc sur la mémoire entendue au sens large, le réservoir culturel commun à l'orateur et à son auditoire.

Outre ces images de mémoire qui agissent comme des coups de projecteur sur la ligne chronologique et qui confèrent à certains événements un caractère mythique, le discours fait usage d'une autre stratégie permettant de réveiller le sentiment patriotique. Il s'agit de la figure de l'analogie. Dans l'épilogue, Obama choisit en effet de faire référence à une tradition importée d'Europe et partagée par la communauté des pêcheurs du golfe: "The Blessing of the Fleet» (1. 183-194). Le président explique que cette prière, devenue cérémonie, est prononcée au début de la saison de la pêche à la crevette afin de les marins qui partent en mer (l'adjectif «safety » est de nouveau prononcé). Et la cérémonie a lieu quoi qu'il advienne. Obama extrait alors du texte d'origine des segments qui peuvent s'appliquer non pas 
seulement au marin qui part en mer mais aussi à tout citoyen américain qui sait faire preuve de force, de courage et de résilience dans une foi inébranlable face aux événements qui se présentent à lui (1. 196-99). Afin de démanteler le mécanisme de l'analogie, nous avons recherché le texte de la prière dans son intégralité :

May God in Heaven fulfill abundantly the prayers which are pronounced over you and your boats and equipment on the occasion of the Blessing of the Fleet. God bless your going out and coming in ; The Lord be with you at home and on the water. May he accompany you when you start on your many journeys : May he fill your nets abundantly as a reward for your labor ; And may he bring you all safely in, when you turn your boats homeward to shore... Amen. ${ }^{9}$

D'un point de vue cognitif, les correspondances établies par l'analogie reposent sur l'activation de deux domaines d'expérience : celui de la pêche (le domaine source) et celui de la vie sur le sol américain (le domaine cible, à avoir le sujet dont l'énonciateur souhaite traiter). Les citoyens deviennent alors des pêcheurs de crevettes, leur vie face aux catastrophes auxquelles ils seront confrontés étant semblable à celle d'un marin qui se retrouve seul avec son bateau pour braver les dangers de la mer. Notons que l'analogie, combinée à l'image de la main de Dieu et à l'emploi métaphorique du substantif «storm », se poursuit jusqu'à la dernière phrase du discours qui précède les remerciements : «And we pray that a hand may guide us through the storm towards a brighter day. » (1. 200-201). La dimension explicitement religieuse du discours ne serait, à ce jour, pas transposable dans un discours prononcé par un président de la République française. Comme démontré, l'analogie à l'étude permet d'insérer une morale au sein de l'épilogue qui est, par définition, «le domaine propre du pathétique »(Gardes-Tamine 1996, 101). Mais ne pouvons-nous également envisager les références faites au texte de cette prière par le président comme un chant patriotique qui permet, dans l'union face à cette crise, de transmettre un message offensif à ses compatriotes?

\section{Le parcours argumentatif du rhéteur : du « plan de bataille » au discours écologique}

Comme l'indique le diagramme présenté plus haut, la deuxième partie la plus longue du discours a été, quelque peu à la hâte, intitulée «ecospeak». Le terme renvoie, lato sensu, aux discours qui portent sur les politiques environnementales, sur la crise énergétique et sur celle qui a été provoquée par le réchauffement climatique. Les deux crises ne sont d'ailleurs pas forcément

9 http://blessingofthefleet.com/ 
présentées comme étant reliées. Un homme politique peut choisir de faire état de la crise énergétique en évoquant, éventuellement et accessoirement, les dommages causés sur l'environnement par des énergies non renouvelables telle que le pétrole-et la pollution engendrée par les déplacements motorisés. Cette évocation peut être exploitée de deux façons au moins dans un raisonnement discursif : (i) les énergies fossiles doivent être remplacées par des énergies renouvelables dans l'objectif de protéger la planète, (ii) la hausse du prix du baril de pétrole crée une crise énergétique dont il faut se dégager au plus vite afin de retrouver une indépendance énergétique qui permette aux humains de continuer à se déplacer à leur guise. Ce dilemme une fois soulevé, il semble toutefois que certains discours politiques tenus sur la crise énergétique n'entrent pas exactement dans la catégorie de l'ecospeak.

\section{Glissement sémantique : de «cleanup » à «clean»}

Le mot qui permet au président de relier son plan de bataille à cette partie est «clean». En effet, et cela n'a rien de surprenant, l'opération de nettoyage évoquée dans le plan de bataille est décrite par le biais de ce terme qui, combiné à «up», est tantôt verbe à particule, tantôt, par dérivation, substantif :

11. To clean up the oil ; the cleanup ; the largest environmental cleanup effort ; cleanup the oil, clean beaches...

Il semble, dans la transcription tout au moins, que les formes «clean up » (donnée officiellement comme verbe) et «cleanup (donnée généralement comme substantif) soient totalement interchangeables, comme si les frontières entre forme et partie de discours se dissolvaient au fil du discours, faisant place au concept de «grand nettoyage ». Notons, au passage, l'écart sémantique entre les verbes «clean» et «clean up». Si l'un renvoie simplement à la notion de nettoyage de la saleté, l'autre fait référence à un programme intensif et radical d'assainissement ou de dépollution.

Le raisonnement par induction permet à Obama de présenter, dans le deuxième temps du discours, la marée noire comme un exemple ou plus exactement comme une leçon pour les États-Unis : «One lesson we've learned from this spill (...)» (1. 117), «But a larger lesson is that (...)» (1. 119). Afin d'élargir le champ de sa réflexion, le président glisse de «cleanup » (le programme de nettoyage) à «clean » (l'adoption des énergies propres) par le biais de la formule, répétée par deux fois, et dont nous avons déjà fait état dans nos travaux : «addiction to fossil fuels » (1. 127 et 1. 164). Cette expression a vu le jour dans le Discours sur l'état de l'Union prononcé par le Président G. W. Bush en 2006 et a été reprise par Obama à maintes occasions pendant sa 
campagne présidentielle contre le candidat républicain McCain (Bonnefille 2009). Sa puissance évocatrice, et qui a choqué bon nombre de démocrates et de républicains lors de sa création ${ }^{10}$, repose sur un système de correspondances issu d'une projection métaphorique entre deux domaines : la réalité quotidienne d'un drogué qui structure celle d'un pays consommateur de pétrole (voir les travaux de Bonnefille 2008 et 2009 pour une analyse détaillée de la métaphore).

Force est de constater que l'image de « l'or noir » ${ }^{11}$ est progressivement détrônée par celle de la substance toxique qui rend le pays malade. Cette source d'énergie, au même titre que toutes les énergies fossiles, devient alors - mais implicitement - «sale » par opposition aux énergies non fossiles, renouvelables ou dites «propres », comme illustré par les expressions suivantes :

12. Clean energy jobs, a clean energy future, clean energy industry, etc.

Et finalement, les discours sur l'énergie articulés autour de l'idée de grand nettoyage et de quête de propreté, voire de pureté12, en temps de crise énergétique et financière ne possèdent-ils pas une part tout aussi fantasmatique dans l'esprit de l'auditoire que l'idée de richesse en des temps où le pétrole coulait à flot aux États-Unis ${ }^{13}$ ?

\section{Un ton ferme : binarité et radicalité}

Si la partie intitulée «plan de bataille » montre de la compassion au fil du discours, le ton d'Obama se durcit. D'abord envers la corruption de la société Minerals Management Service (1. 104 et 1. 111). Ensuite, envers le manque de sécurité et les dangers impliqués par ce type de forages en eaux profondes. Puis face à la crise énergétique que traverse le pays. Les figures de la radicalité entrent alors en scène :

13. An entire way of life being threatened by a menacing cloud of black crude (1. 13334), Our determination to fight for the America we want for our children (1. 178-79), The consequences of our inaction are in plain sight (1. 130), to embrace a clean energy future (1. 136), our capacity to shape our destiny (1. 177-78), to seize control of our own destiny (1. 140), seize the moment (1. 151).

10 Aussi, à l'inverse d'Obama, McCain a choisi de ne pas employer cette métaphore de l'addiction pendant sa campagne (Bonnefille 2008).

11 Voir, à propos de l'histoire des États-Unis et du pétrole, Le Secret des 7 sept sæurs, 2010, série documentaire diffusée sur France 5.

12 Voir Lakoff 2004 (22-23) au sujet de l'emploi des adjectifs « safe », «clean » et « healthy » par le Parti républicain.

13 De 1986 à 2000 aux États-Unis, comme l'indique le site de la CNUCED, http://unctad.org/infocomm/francais/petrole/prix.htm 
Sur un ton ferme, voire paternaliste ${ }^{14}$, Obama en appelle à présent à la prise de conscience de tout le peuple américain face à la crise énergétique et, plus concrètement, face aux habitudes et aux besoins de la population en matière de consommation d'énergie. Ici, le déterminant possessif «our» et le pronom «we» ne font plus exclusivement référence à l'administration. Par ailleurs, la série de segments ci-dessus indique que le programme moteur de saisie, tant par la main que par le corps tout entier, est sollicité comme domaine source dans plusieurs métaphores qui expriment et rappellent le contrôle et le pouvoir de décision dont le pays peut et doit faire preuve sur le plan énergétique. Dans la même veine, les métaphores conceptuelles dorénavant classiques (voir Bonnefille 2011b) du déplacement dans l'espace sont également à l'œuvre :

14. to embark on a national mission (1. 137), The path forward has been blocked (1. 12829), Move our country towards energy independence (1. 155).

Le schème mental du CHEMIN (Johnson 1987) est projeté sur l'évolution par laquelle doit passer le pays : de la consommation d'énergies fossiles à celle d'énergies renouvelables.

L'usage répété de formes comparatives et superlatives constitue un autre moyen de raffermir le ton. En effet, les mécanismes comparatifs induisent, d'un point de vue syntactico-sémantique, un mouvement binaire, donc élémentaire et aisé d'accès pour l'auditoire, entre deux entités. Et, en définitive, ce qui se trouve à gauche dans la formule, plus ou moins explicitement d'ailleurs, est systématiquement et tout simplement moins valorisé que ce qui se trouve à droite :

15. One of the lessons we've learned (...) but a larger lesson is that (...), drilling for oil these days entails greater risk, something better awaits for us, that a hand may guide us through the storm towards a brighter day.

Une analyse plus fine de ce mouvement binaire, et donc simplificateur, devrait inclure les segments contenant les adverbes «ever», «never», «always », «already », associés à l'aspect HAVE + -EN. La valeur de bilan qui découle de ces associations peut prendre, elle aussi, un tour radical (une face blanche et une face noire, en quelque sorte) qui ne reflète pas forcément la complexité d'une réalité qui est rarement binaire :

16. Because there has never been a leak of this size at this depth, stopping it has tested the limits of human technology. (1. 11-12).

14 Faute de place, nous ne pouvons nous arrêter sur l'emploi fréquent des formules performatives et sur celles qui soulignent l'action politique associées au pronom «I $\mathrm{I}$, telles que : «I assembled a team », «I have issued a six-month moratorium », «I have established », etc. 
Les formes superlatives fonctionnent de manière identique, bien sûr, même si l'élément de gauche n'apparaît pas clairement :

17. Our nation's best scientists, the worst environmental disaster, the largest environmental cleanup effort in our nation's history, the most painful and powerful reminder, the oil spill is not the last crisis America will face.

Dans ce type de discours, comme, du reste, dans le discours publicitaire, ces formes superlatives ont pour fonction première de permettre à l'élément concerné («environmental disaster», «environmental cleanup », etc.) d'atteindre le degré ou la puissance sémantique maximums. Sur le plan cognitif, l'auditoire est ainsi plongé dans un état de saturation sémantique (au sens logique du terme $^{15}$ ) qui ne laisse aucune place à la nuance dans l'espace métaphorique de réception du message.

\section{Conclusion}

Les outils de la rhétorique et sa terminologie alliés à l'analyse de processus cognitifs issus de la recherche en linguistique cognitive tels que le cadrage narratif, les mécanismes d'analogie et les métaphores conceptuelles, la projection de schèmes mentaux ou de scenarii moteurs sur des domaines cibles particuliers, les images de mémoire et leur importance dans la construction d'une représentation, etc. confèrent à l'analyse de discours une dimension nouvelle quant à l'exploration de la construction d'une représentation par un orateur et du sens qu'elle est censée véhiculer auprès de l'auditoire. C'est en tous les cas vers cet objectif que doit tendre la discipline de la rhétorique cognitive telle que nous l'avons définie par ailleurs.

Dans ce cas précis - la marée noire du golfe - rappelons que bien plus que le réchauffement climatique, la crise énergétique, sur fond de récession économique, se trouve au cœur des préoccupations du gouvernement et des citoyens américains et qu'elle nécessite donc d'être représentée de manière stratégiquement adéquate.

La première partie du discours repose clairement sur des mécanismes de storytelling qui plaquent «sur la réalité des récits artificiels, bloque[nt] les échanges, sature[nt] l'espace symbolique (...) » (Salmon 2007, 16-17). Dans un second temps, en privilégiant un mode de représentation binaire ${ }^{16}$ simpliste tel que nous l'avons décrit dans la deuxième grande partie de cet article, Obama

15 «Saturation : LOG. Caractère d'un système axiomatique où l'on ne peut adjoindre un nouvel axiome sans qu'il en résulte une théorie contradictoire. » (Le Petit Larousse 2009).

16 Le discours prononcé par le président Sarkozy aux Nations Unies en 2009 reposait essentiellement sur ce mécanisme, alors que celui du président Obama faisait preuve d'une nuance et d'une souplesse plus marquées (Bonnefille 2011b). 
applique à la lettre les stratégies de l'art de persuader dans une perspective d'identité énonciative radicale. Si nous le comparons à celui que nous avons décrit dans nos travaux précédents (Obama en campagne contre McCain au cours de l'été 2008 et Obama au sommet des Nations Unies en 2009), l'éthos du président apparaît en effet plus rigide. 


\section{Bibliographie}

\section{Corpus}

http://www.whitehouse.gov/the-press-office/remarks-president-nation-bp-oil-spill

\section{Ouvrages cités et consultés}

ARISTOTE, 1991. Rhétorique, Paris, Le Livre de Poche.

BONNEFILLE, Stéphanie, 2008 (27-61). «When green rhetoric and cognitive linguistics meet : President G. W. Bush's environmental discourse in his State of the Union Addresses (2001-2008)»,

Metaphorik.de.http://www.metaphorik.de/index.htm

2009 (39-67). «Conceptualisation de la question environnementale aux États-Unis : les mots verts des candidats Obama et McCain », Bulletin de la Société de Stylistique Anglaise 32.

2011b (145-162). «A cognitive rhetoric approach to two different political speeches: Obama and Sarkozy's remarks at the U.N.'s climate change summit (2009) », Anglophonia 30, Toulouse, Presses Universitaires du Mirail.

CAILloIs, Roger, 1958. Art poétique, Paris, Gallimard.

Cox, Robert, 2006. Environmental Communication and the Public Sphere, Thousand Oaks, Sage Publications.

DARBYSHIRE, Alec Edward, 1971. A Grammar of Style, London, A. Deutsch.

Fromilhague, Catherine, 2010. Les Figures de style, Paris, Armand Colin.

Fromilhague, Catherine et SANCIER, Anne, 1991. Introduction à l'analyse stylistique, Paris, Bordas.

GARDES-TAMINE, Joëlle, 1996. La Rhétorique, Paris, Armand Colin.

GreEnBAum, Sidney et QUIRK. Randolph, 1990. A Student's Grammar of the English Language, Harlow, Essex, Longman.

Johnson, Mark, 1987. The Body in the Mind, Chicago \& London, University of Chicago Press.

KILlingsworth, Jimmie M. \& PALMER, Jacqueline S., 1995. Ecospeak. Rhetoric and Environmental Politics in America, Carbondale, Southern Illinois University Press. 
LAKOFF, George, 1980. Metaphors We Live By, Chicago \& London, University of Chicago Press. 1996. Moral Politics, Chicago \& London, University of Chicago Press. 2004. Don't Think of an Elephant, White River Junction, Chelsea Green Publishing Company.

Maingueneau, Dominique, 1996. Les Termes clés de l'analyse du discours, Paris, Seuil.

NORRIS, Pippa, Kern, Montague et Just, Marion (eds), 2003. Framing Terrorism: The News Media, the Government, and the Public, New York \& London, Routledge.

RITAINE, Evelyne, 2010 (201-221). «Dramaturgie de l'intrusion migratoire : teatro all'italiana », D.-C. Martin (dir.) L'Identité en jeux : Pouvoirs, identifications, mobilisations, Paris, Karthala.

SALMON, Christian, 2007. Storytelling, la machine à fabriquer des histoires et à formater les esprits, Paris, La découverte.

SEMINO, Elena \& CULPEPER Jonathan (eds), 2002. Cognitive Stylistics: Language and Cognition in Text Analysis, Amsterdam \& Philadelphia, John Benjamins Publishing Company.

SALBAYRE, Sébastien \& VINCENT-ARNAUD, Nathalie, 2006. L'Analyse stylistique : Textes littéraires de langue anglaise, Toulouse, Presses universitaires du Mirail.

Suhamy, Henri, 1981. Les Figures de style, Paris, Presses universitaires de France.

TISSET, Carole, 2000. Analyse linguistique de la narration, Paris, Sedes.

ToOLAN, Michael, 1988. Narrative: A Critical Linguistic Introduction, London, Routledge.

TURNER, Mark, 1988 (3-23). «Categories and Analogies », Analogical Reasoning : Perspectives of Artificial Intelligence, Cognitive Science, and Philosophy, Dordrecht \& Boston \& London, Kluwer Academic Publishers.

\section{Sites consultés, documentaires, magazines, dictionnaires}

«Les pensées vertes », 2010. Les Grands dossiers des sciences humaines, n 19 , juinjuillet-août.

Le Secret des 7 sept sœurs, 2010. Série documentaire, France 5 (épisode ${ }^{\circ} 1,9$ octobre 2011), producteurs Arnaud Hamelin et Frédéric Tonolli, France Télévision \& Sunset Presse.

http://blessingofthefleet.com/

http://unctad.org/infocomm/francais/petrole/prix.htm

Le Petit Larousse 2010, Paris, Larousse. 